Research Article

\title{
An Improved Nonhomogeneous Grey Model with Fractional-Order Accumulation and Its Application
}

\author{
Shuanghua Liu, ${ }^{1}$ Qin Qi ${ }^{2},{ }^{2}$ and Zhiming $\mathrm{Hu}^{3}$ \\ ${ }^{1}$ School of Mathematics and Statistics, Baise University, Baise 533000, China \\ ${ }^{2}$ School of Economics and Management, Tongji University, Shanghai 200092, China \\ ${ }^{3}$ Zhejiang College, Shanghai University of Finance \& Economics, Jinhua 321013, China \\ Correspondence should be addressed to Qin Qi; qiqin_jxsh@163.com
}

Received 31 March 2021; Revised 14 May 2021; Accepted 4 June 2021; Published 21 June 2021

Academic Editor: Lifeng Wu

Copyright (c) 2021 Shuanghua Liu et al. This is an open access article distributed under the Creative Commons Attribution License, which permits unrestricted use, distribution, and reproduction in any medium, provided the original work is properly cited.

\begin{abstract}
The nonhomogeneous grey model has been seen as an effective method for forecasting time series with approximate nonhomogeneous index law, which has been widely used in diverse disciplines on account of its high prediction precision. However, there remains room for improvements. For this, this study presents an improved nonhomogeneous grey model by incorporating the dynamic integral mean value theorem and fractional accumulation simultaneously. In order to promote the efficacy of the optimised model, we apply the whale optimization algorithm (WOA) to ascertain its optimal parameter. In particular, two examples are conducted to validate the superiority of the proposed model in contrast with other benchmarks, and the experimental results show that the mean absolute percentage error of the proposed approach is $808692 \%$ and $6.0706 \%$, respectively, indicating the proposed approach performs better than other competing models.
\end{abstract}

\section{Introduction}

The prediction plays an important role in management and decision-making science. In the past decades, there are a variety of forecasting technique, such as autoregressive integrated moving average (ARIMA), artificial neural networks, and support vector machine. These models have their modeling mechanism and application scope. However, these models have a common flaw that they usually require more samples to build the model so as to obtain relatively high prediction precision. However, the behaviors of most systems in practice are often uncertain and unknown, and the effect of large-sized sample-based prediction models may be relatively poor. On this basis, grey system models focusing on small-sized observations have been received more attention in the recent decades [1].

As previous literature revealed, for the ability to analyse and process data sequences, grey-based models have been broadly applied among diverse disciplines because of their excellent implementation on small-scale sample modeling, such as natural gas consumption [2-4], electric power supply and demand [5-8], renewable energy $[9,10]$, industry $[11,12]$, and medicine $[13,14]$. Although grey-based models have their own advantages, there still exist some shortcomings. For instance, the traditional grey model (denoted as $\operatorname{GM}(1,1)$ ) only fits time-series sequence with pure exponential characteristics well but fails to fit time series with other features. To this end, Cui et al. [15] brought forward a nonhomogeneous grey model for forecasting data series with approximate nonhomogeneous index feature. In fact, the nonhomogeneous grey model is nascent, and this model has certain defects. To be specific, these defects mainly exist in the background value, cumulative order, time response function, and application scope. Later, to improve the nonhomogeneous grey model, many scholars have paid their attention to this issue. For example, Ma and Liu [16] optimised the background value in accord with general expression for its time response function, also notable is that this optimised model was successful to apply in practice. Tong et al. [17] designed a newly designed nonhomogeneous grey model to simultaneously fit time approximately homogeneous and nonhomogeneous index features. 
Considering the vital impact of the background value on the prediction performance of the nonhomogeneous grey model, Zeng and Liu [18] presented an improved nonhomogeneous grey model based on fractional-order accumulation, which can achieve high accurate prediction by virtue of fractional-order accumulation. Subsequently, a series of variants of the nonhomogeneous grey model with fractional-order accumulation have emerged. For example, $\mathrm{Wu}$ et al. [19] established a discrete nonhomogeneous grey model based on fractional-order accumulation. Later, $\mathrm{Wu}$ et al. [20] presented a conformable fractional nonhomogeneous grey model to predict carbon emissions for BRIC countries. The abovementioned optimization measures have dramatically improved the prediction performance of the existing grey-based model and enriched the grey modeling theory.

Admitted, there exist a variety of nonhomogeneous grey models; however, these approaches are not universal. That is, many optimization approaches are only suitable for special cases. The optimization methods for increasing the prediction precision of the grey models include fractional accumulation and integral median theorem aiming to optimize the background value. Recalling, Wu et al. [21] first placed the fractional accumulation on the grey system models, which is a significant innovation to improve the prediction precision of grey models, on the foundation of grey-based models with fractional-order accumulation [22-25].

To further increase the prediction performance of the abovementioned models, there are a variety of studies that are committed to explore the combination of fractionalorder accumulation and grey modeling technique. For instance, Zhu et al. [26] brought forward a newly designed fractional grey model, of which the fractional accumulated generating operation sequence is dependent on an adaptive grey score weight; additionally, this model is applied to predict Jiangsu's electricity consumption. Chen et al. [27] put forward a fractional Hausdorff grey model, and Ma et al. [28] presented a novel grey model by using conformable fractional-order accumulation. These studies greatly enriched the grey system theory and enabled the combination of fractional calculus with the grey modeling technique. On the other hand, the adaptation of the integral median value theorem for improving the background value can enhance the prediction precision of the grey-based models $[29,30]$.

On the foundation of the previous knowledge, this study constructs a novel discrete nonhomogeneous grey model by incorporating the idea of fractional accumulation and the dynamic integral median theorem; the composite grey model (denoted as $\operatorname{FDNGM}(1,1)$ for short) is developed thereby, which can fit diverse series sequence through altering the fractional accumulation order and backgroundvalue coefficients. The principal innovations and contributions are outlined as follows. (1) We combine the dynamic background value with the grey modeling technique. (2) An effective intelligent technique, namely, the whale optimization algorithm, is utilized to ascertain the proper parameters for the proposed approach. (3) The several examples are used to certify the feasibility of this model.
The rest of this study is listed as follows. Section 2 briefly depicts the computational steps of the basic nonhomogeneous grey model. The proposed approach is studied in Section 3. Section 4 introduces the solution method for the proposed model. Section 5 reports the experimental results, and Section 6 concludes.

\section{Basic NGM( $(1,1, k, c)$ Model}

Assuming a raw sequence can be denoted as $S^{0}=\left\{s^{0}(k) \mid k=1,2, \ldots, n\right\}, n \geq 4$, whose 1 st-order accumulative generation operator data series is calculated as

$$
S^{1}=\left\{s^{1}(i) \mid i=1,2, \ldots, n\right\},
$$

where $s^{1}(i)=\sum_{j=1}^{i} s^{0}(j), i=1,2, \ldots, n$.

Then, the differential equation for the basic $\operatorname{NGM}(1,1, k, c)$ model is written as

$$
\frac{\mathrm{d} s^{1}(t)}{\mathrm{d} t}+a s^{1}(t)=b t+c .
$$

For the sake of estimating the system parameters for the abovementioned model, we get the discrete formula for equation (2) as

$$
s^{0}(i)+a z^{1}(i)=b \frac{i^{2}-(i-1)^{2}}{2}+c .
$$

In equation (3), $z^{1}$ refers to the background value, for which $z^{1}(i)=0.5\left(s^{1}(i)+s^{1}(i-1)\right)$.

With the help of the least-squares method, the system parameters for the conventional $\operatorname{NGM}(1,1, k, c)$ model should be computed as

$$
(\widetilde{a}, \tilde{b}, \widetilde{c})^{T}=\left(\gamma^{T} \gamma\right)^{-1} \gamma^{T} \Omega
$$

where

$$
\begin{aligned}
\gamma & =\left(\begin{array}{ccc}
-z^{1}(2) & \frac{3}{2} & 1 \\
-z^{1}(3) & \frac{5}{2} & 1 \\
\vdots & \vdots & \vdots \\
-z^{1}(n) \frac{\left(n^{2}-(n-1)^{2}\right)}{2} & 1
\end{array}\right) \\
\Omega & =\left(\begin{array}{c}
s^{0}(2) \\
s^{0}(3) \\
\vdots \\
s^{0}(n)
\end{array}\right)
\end{aligned}
$$

After that, we get the time response function for equation (2) calculated as 


$$
\begin{aligned}
s^{1}(i)= & \left\{s^{0}(1)+\frac{b-a c-a b}{a^{2}}\right\} \exp (-a(k-1)) \\
& +\frac{b k}{a}+\frac{(a c-b)}{a^{2}} .
\end{aligned}
$$
by

Eventually, the restored values of the raw data are given

$$
\tilde{x}^{(0)}(k)= \begin{cases}\tilde{x}^{(1)}(k)-\tilde{x}^{(1)}(k-1), & k=2,3, \ldots, \\ \tilde{x}^{(1)}(1), & k=1 .\end{cases}
$$

We observe from the modeling procedure above that the prediction precision is dependent on the system parameters influenced by the background value and cumulative sum operator. In $\operatorname{NGM}(1,1, k, c)$, we apply the integer-order accumulation and trapezoid formula to generate the accumulated sequence and background value, and it is evident that the fixed accumulation order and approximate discrete error will impair the prediction performance to a large degree. For this, we apply the fractional accumulation and dynamic integral median theorem on the modeling procedure for improving the prediction capacity of the existing nonhomogeneous model.

\section{Presentation of FDNGM( $1,1, k, c)$}

Suppose that

$$
S^{0}=\left\{s^{0}(i) \mid i=1,2, \ldots, n\right\},
$$

is the given data sequence that is nonnegative, then its r-order fractional accumulating generation operation data series can be calculated as

$$
S^{r}=\left\{s^{r}(i) \mid i=1,2, \ldots, n\right\},
$$

where $s^{r}(i)==\sum_{j=1}^{i}\left(\begin{array}{c}i-j+r-1 \\ i-j\end{array}\right) s^{0}(j), i=1,2, v, n$.

Based on $S^{r}$, the differential equation for the proposed model is obtained as

$$
\frac{\mathrm{d} s^{r}(t)}{\mathrm{d} t}+a s^{r}(t)=b t+c .
$$

In this study, we place the dynamic integral median theorem on the background value for the proposed model so as to eliminate the discretization error generated by the transition process.

Theorem 1. If $s^{r}(j)$ is continuous over given interval $[i-1, i]$, then we get

$$
\int_{i-1}^{i} s^{r}(j) \mathrm{d} j=s^{r}(I)=(1-\varphi) s^{r}(i-1)+\varphi s^{r}(i),
$$

where $\varphi \in[0,1]$ and $I \in[i-1, i]$.

Proof. Since $s^{r}(j)$ keeps continduous over $[i-1, i]$, there exist the maximum value $D$ and minimum value $d$ for $s^{r}(j)$ over $[i-1, i]$, which makes $d \leq s^{r}(j) \leq D$ hold true; then, we have

$$
\varphi d \leq \varphi s^{r}(i) \leq \varphi D
$$

which is also

$$
(1-\varphi) d \leq(1-\varphi) s^{r}(i-1) \leq(1-\varphi) D .
$$

Combining equations (12) and (13), we have

$$
d \leq(1-\varphi) s^{r}(i-1)+\varphi s^{r}(i) \leq D .
$$

In accord with the intermediate value theorem, $\phi \in[k-$ $1, k]$ that makes $s^{r}(\phi)=(1-\varphi) s^{r}(i-1)+\varphi s^{r}(i)$ hold. It can be further concluded that for any value $s^{r}(\tilde{\phi})$ of the continuous function $s^{r}(i)$ on $[i-1, i]$, there exists $\varphi_{1} \in[0,1]$ that makes $s^{r}(\tilde{\phi})=\left(1-\varphi_{1}\right) s^{r}(i-1)+\varphi_{1} s^{r}(i)$ hold true. Thereafter, in accord with the integral mean value theorem, we get

$$
\int_{i-1}^{i} s^{r}(j) \mathrm{d} j=s^{r}(I), \quad I \in[i-1, i] .
$$

Furthermore, we have

$$
\int_{i-1}^{i} s^{r}(j) \mathrm{d} j=s^{r}(I)=(1-\varphi) s^{r}(i-1)+\varphi s^{r}(i) .
$$

It is proved.

Therefore, the newly designed background value for the proposed model can be given as

$$
\widetilde{z}^{r}(i)=\left(1-\varphi_{1}\right) s^{r}(i-1)+\varphi_{1} s^{r}(i), \quad i=2,3, \ldots, n .
$$

Then, we get the least-squares estimation for system parameters expressed as

$$
(\tilde{a}, \tilde{b}, \widetilde{c})^{T}=\left(\vartheta^{T} \vartheta\right)^{-1} \vartheta^{T} \omega,
$$

where

$$
\begin{aligned}
& \vartheta=\left(\begin{array}{ccc}
-\widetilde{z}^{r}(2) & \frac{3}{2} & 1 \\
-\widetilde{z}^{r}(3) & \frac{5}{2} & 1 \\
\vdots & \vdots & \vdots \\
-\widetilde{z}^{r}(n) \frac{\left(n^{2}-(n-1)^{2}\right)}{2} & 1
\end{array}\right), \\
& \omega=\left(\begin{array}{c}
s^{r-1}(2) \\
s^{r-1}(3) \\
\vdots \\
s^{r-1}(n)
\end{array}\right) .
\end{aligned}
$$

Similar to the calculation steps mentioned in Section 2, the time response function for the proposed model can be acquired. Moreover, the predicted values of the original data series is given as 


$$
\widetilde{s}^{0}(i)=\left\{\alpha^{1} \widetilde{s}^{r}(1), \alpha^{1} \widetilde{s}^{r}(2), \ldots, \alpha^{1} \widetilde{s}^{r}(n)\right\}
$$

\section{Determination of Parameter}

Notice that the proposed model is constructed under the assumption that the emerging coefficients $\varphi_{1}, r$ are known.
Thus, they have a significant impact on the prediction precision directly. Aiming to effectively enhance the prediction precision of the proposed model, a relative simple optimization problem can be established, whose calculation formula can be defined as

$$
\begin{aligned}
& \min f\left(r, \varphi_{1}\right)=\frac{1}{n-1} \sum_{i=2}^{n} \frac{\left|\widetilde{s}^{r}(i)-s^{r}(i)\right|}{s^{r}(i)} \times 100 \% \\
& \left\{\begin{array}{l}
\varphi_{1} \in[0,1], \quad r \geq 0 \\
(\widetilde{a}, \widetilde{b}, \widetilde{c})=\left(\vartheta^{T} \vartheta\right)^{-1} \vartheta^{T} \omega,
\end{array}\right. \\
& \vartheta=\left(\begin{array}{ccc}
-\widetilde{z}^{r}(2) & \frac{3}{2} & 1 \\
-\widetilde{z}^{r}(3) & \frac{5}{2} & 1 \\
\vdots & \vdots & \vdots \\
-\widetilde{z}^{r}(n) \frac{\left(n^{2}-(n-1)^{2}\right)}{2} & 1
\end{array}\right), \\
& \omega=\left(\begin{array}{c}
s^{r-1}(2) \\
s^{r-1}(3) \\
\vdots \\
s^{r-1}(n)
\end{array}\right), \\
& s^{1}(i)=\left\{s^{0}(1)+\frac{b-a c-a b}{a^{2}}\right\} \exp (-a(k-1))+\frac{b k}{a}+\frac{(a c-b)}{a^{2}} .
\end{aligned}
$$

As previous literature revealed, the abovementioned equation is difficult to solve by the ordinary approach on account of its nonlinear features. Therefore, this study introduces a metaheuristic technique, namely, the whale optimization algorithm (denoted as WOA), to search the best values of the background-value coefficients and fractional accumulation order.

In 2016, Mirijalili and Lewis [31] designed the whale optimization algorithm which describes the social behavior of the whale group. Over the past 5 years, this algorithm has been applied in various fields [32, 33]. More importantly, this approach is regarded as an effective technique to solve nonlinear optimization problems [34]. This is the main motivation for us to choose the WOA to solve the abovementioned equation. To be specific, the modeling steps of the WOA are listed.

Whales move in spiral to surround the school of fish, which is considered the best solution for predation. After that, they change their position by reference to the candidate solution. We get the expression of this behavior shown as 


$$
\vec{P}(i+1)= \begin{cases}\vec{P}^{*}(i)-(2 f(i) \vec{r}-f(i)) \vec{D}, & \xi<0.5(a), \\ \left|\vec{P}^{*}(i)-\vec{P}(i)\right| e^{\beta i} \cos (2 \pi l)+\vec{P}^{*}(i), & \xi \geq 0.5(b) .\end{cases}
$$

In equation (22), $\vec{P}(i), \vec{P}^{*}(i)$ denote the whales' current position and their current best position, respectively. $\vec{r}$ refers to a arbitrary number generated within $[0,1], l$ is a random number produced from $[-1,1], \beta$ is the key for controlling the shape of whales' moving spiral, $T$ is denoted as the maximum number of iterations, and the movement strategy will be chosen by the probability $\xi$. Whales change their position according to the equation expressed as

$$
\vec{P}(i+1)=\vec{P}_{r}(i)-\vec{C}\left|2 \vec{P}_{r}(i) \vec{r}-\vec{P}(i)\right| .
$$

In equation (23), $\vec{p}_{r}(i)$ denotes the position of random whale in the group. Additionally, we define the fitness function for calculating the fitness of each whale expressed as

$$
\text { fitness }=\frac{1}{n-1} \sum_{i=2}^{n}\left|\frac{\widetilde{s}^{0}(i)-s^{0}(i)}{s^{0}(i)}\right| \times 100 \% .
$$

The flowchart of the proposed model based on the WOA technique is shown in Figure 1.

\section{Application}

This section conducts two real cases to demonstrate the superiority of the proposed approach in contrast with other benchmark models. The models used for comparison include the polynomial regression model (PR) proposed by literature [35], the FHGM $(1,1)$ model proposed by literature [26], and the $\operatorname{ONGM}(1,1, k, c)$ model proposed by literature [36].

Case 1. (Prognosticating Chongqing's electric power consumption) With the depletion of nonrenewable energy sources such as coal, crude oil, and nuclear energy, electrical energy is becoming more and more important in our lives and production. Overestimation and underestimation of short-term electricity consumption will waste the energy or cause unnecessary loss. Therefore, accurate and effective forecasting of electricity consumption can help power system operators and market participants to propose bidding strategies and ensure consumer's electricity supply based on corresponding forecast information, thereby reducing the cost of electricity consumption and reducing energy consumption.

The original data were gathered from the Statistical Yearbook of China. Regarding the calculation procedure, first, the searching process by the WOA is given in Figure 2; accordingly, all the system parameters are listed in Table 1. By reference with this study and references therein, the predicted values and error-value metrics generated by the competing models are given in Tables 2 and 3, respectively. What is more, the error distribution is shown in Figure 3.

In accord with the predicted values mentioned in $\mathrm{Ta}$ ble 2 , we find that the predicted values by the $\operatorname{NGM}(1,1, k, c)$, $\operatorname{FHGM}(1,1)$, and $\operatorname{ONGM}(1,1, k, c)$ models all deviate far away from the actual series, and those of the proposed approach are much closer to the actual data. In addition, we observe from Table 3 that the MAPE values of the $\operatorname{FDNGM}(1,1, k, c)$ model are obviously lower than those of others. Moreover, the same discovery can be supported in Figure 3, indicating the proposed approach is more suitable for predicting the electric power consumption in Chongqing than other competitors.

Case 2. (Forecasting China's natural gas consumption) Chinese economy experienced a high growth in the new stage; additionally, natural gas, as a clean energy, has been regarded as an alternative source. Under the background of oil and gas system reform, the supply and demand for natural gas have dramatically changed as time goes on. Developing a accurate and sustainable forecasting model for the development trend of natural gas is of great practical significance.

Analogous with Case 1, the original series is gathered from Statistical Yearbook of China, as given in Table 4. First, the searching process by the WOA technique-based proposed approach is shown in Figure 4. By referring to the current study and references therein, we tabulate the predicted values of China's natural gas consumption and corresponding error-value indices by using different models in Tables 4 and 5, respectively.

By screening the predicted values of natural gas consumption in Table 5 that the predicted values of $\operatorname{NGM}(1,1, k, c), \operatorname{FHGM}(1,1)$, and $\operatorname{ONGM}(1,1)$ models deviate away from the observations, and the those of the proposed approach are much closer to the actual data. What is more, the error of the proposed model is smaller than other benchmarks, as shown in Figure 5. Specifically, the APEs and MAPEs of the proposed approach in this case, referring that the proposed model, should be regarded as a promising approach in this case. 


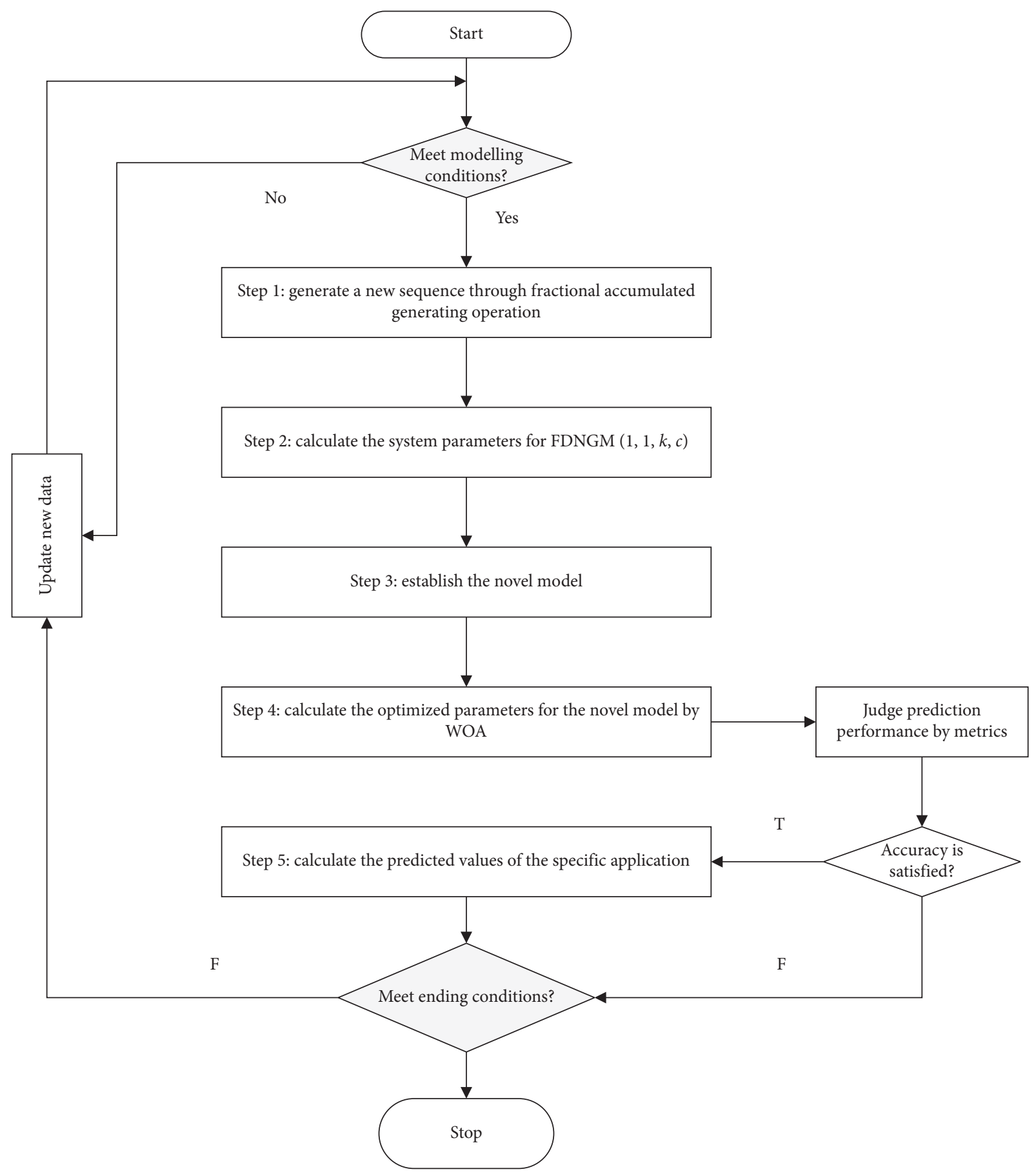

FIgURE 1: Schematic diagram of the calculation process of the $\operatorname{FDNGM}(1,1, k, c)$ model. 


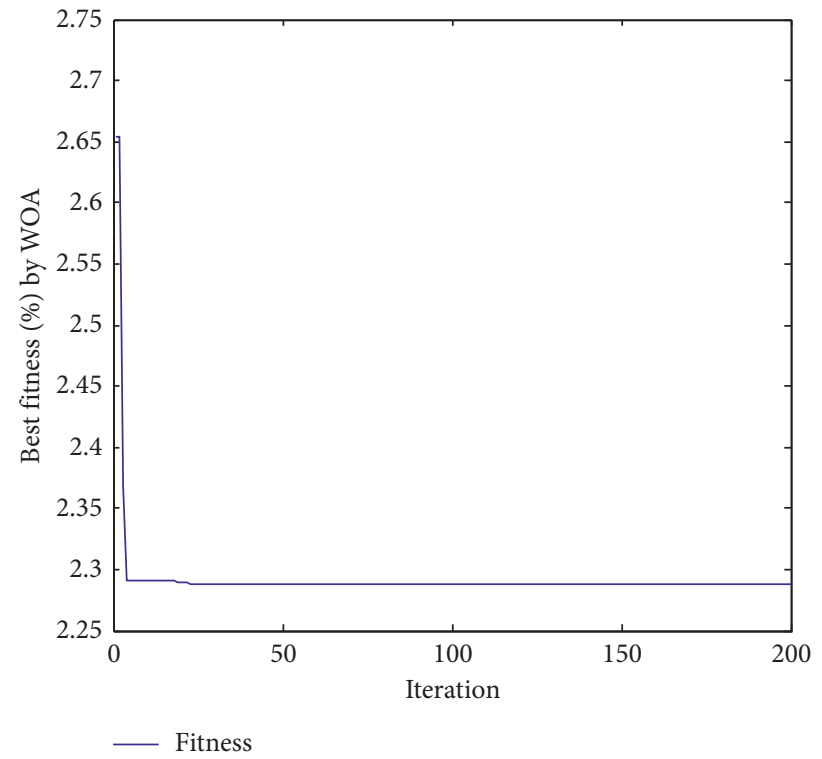

Figure 2: Searching process of the WOA-based $\operatorname{FDNGM}(1,1, k, c)$ model in Case 1.

TABLE 1: Parameters of the competitors in the two real-world cases.

\begin{tabular}{|c|c|c|c|c|c|c|c|c|}
\hline Parameter & \multicolumn{4}{|c|}{ Case 1} & \multicolumn{4}{|c|}{ Case 2} \\
\hline$\varphi_{1}$ & \multicolumn{4}{|c|}{0.85363} & \multicolumn{4}{|c|}{0.98361} \\
\hline$\varphi_{2}$ & \multicolumn{4}{|c|}{0.69486} & \multicolumn{4}{|c|}{0.99217} \\
\hline$\varphi_{3}$ & \multicolumn{4}{|c|}{0.62772} & \multicolumn{4}{|c|}{0.70541} \\
\hline$\varphi_{4}$ & \multicolumn{4}{|c|}{0.49884} & \multicolumn{4}{|c|}{0.99948} \\
\hline$\varphi_{5}$ & \multicolumn{4}{|c|}{0.66898} & \multicolumn{4}{|c|}{0.75600} \\
\hline$\varphi_{6}$ & \multicolumn{4}{|c|}{0.10179} & \multicolumn{4}{|c|}{0.03251} \\
\hline$\varphi_{7}$ & \multicolumn{4}{|c|}{0.02099} & \multicolumn{4}{|c|}{0.98822} \\
\hline$\varphi_{8}$ & \multicolumn{4}{|c|}{0.63933} & \multicolumn{4}{|c|}{0.96816} \\
\hline$\varphi_{9}$ & \multirow{2}{*}{\multicolumn{4}{|c|}{$\begin{array}{l}0.37460 \\
0.28715\end{array}$}} & \multirow{2}{*}{\multicolumn{4}{|c|}{$\begin{array}{l}0.99913 \\
0.02580\end{array}$}} \\
\hline$r$ & & & & & & & & \\
\hline- & NGM & FHGM & FDNGM & ONGM & NGM & FHGM & FDNGM & ONGM \\
\hline$A$ & -0.06433 & -0.05620 & 0.00113 & -0.05620 & -0.12639 & -0.10767 & 0.10902 & -0.10767 \\
\hline$B$ & 22.33115 & 26.88054 & 4.67769 & 26.88054 & 21.76010 & 35.34812 & 25.82133 & 35.34812 \\
\hline C & 244.6430 & 237.2880 & 90.1820 & 237.2880 & 193.2125 & 160.5392 & 16.92224 & 160.5392 \\
\hline
\end{tabular}

TABLE 2: The predicted values of original domain by employing the competing models in Case 1.

\begin{tabular}{|c|c|c|c|c|c|c|}
\hline Year & Data & NGM & $\operatorname{FHGM}(1,1)$ & FDNGM & $\mathrm{PR}(3)$ & ONGM \\
\hline 2003 & 294.19 & 294.19 & 294.83 & 294.19 & 294.09 & 294.19 \\
\hline 2004 & 309.06 & 306.71 & 302.47 & 305.74 & 314.59 & 302.43 \\
\hline 2005 & 347.68 & 350.15 & 347.61 & 348.78 & 347.42 & 347.57 \\
\hline 2006 & 405.20 & 396.48 & 395.35 & 398.12 & 390.42 & 395.31 \\
\hline 2007 & 449.22 & 445.90 & 445.86 & 450.10 & 441.44 & 445.81 \\
\hline 2008 & 484.41 & 498.59 & 499.28 & 503.75 & 498.32 & 499.23 \\
\hline 2009 & 533.80 & 554.79 & 555.79 & 558.71 & 558.93 & 555.74 \\
\hline 2010 & 626.44 & 614.72 & 615.57 & 614.86 & 621.10 & 615.52 \\
\hline 2011 & 717.03 & 678.63 & 678.81 & 672.13 & 682.68 & 678.75 \\
\hline 2012 & 723.50 & 746.79 & 745.70 & 730.52 & 741.53 & 745.64 \\
\hline 2013 & 813.30 & 819.48 & 816.46 & 790.01 & 795.49 & 816.40 \\
\hline 2014 & 867.24 & 897.00 & 891.30 & 850.59 & 842.41 & 891.24 \\
\hline 2015 & 875.37 & 979.67 & 970.48 & 912.28 & 880.13 & 970.41 \\
\hline 2016 & 924.89 & 1067.83 & 1054.23 & 975.06 & 906.51 & 1054.16 \\
\hline 2017 & 996.55 & 1161.85 & 1142.82 & 1038.95 & 919.40 & 1142.75 \\
\hline 2018 & 1118.79 & 1262.12 & 1236.54 & 1103.93 & 916.64 & 1236.46 \\
\hline
\end{tabular}


TABLE 3: The error-value indices of the competing models in Case 1.

\begin{tabular}{|c|c|c|c|c|c|c|}
\hline Year & Data & NGM (\%) & FHGM (\%) & FDNGM (\%) & $\operatorname{PR}(3)$ & ONGM (\%) \\
\hline 2003 & 294.19 & 0.0000 & 0.2159 & 0.0000 & 0.0332 & 0.0000 \\
\hline 2004 & 309.06 & 0.7614 & 2.1318 & 1.0743 & 1.7908 & 2.1437 \\
\hline 2005 & 347.68 & 0.7111 & 0.0210 & 0.3173 & 0.0745 & 0.0322 \\
\hline 2006 & 405.20 & 2.1508 & 2.4305 & 1.7479 & 3.6479 & 2.4406 \\
\hline 2007 & 449.22 & 0.7399 & 0.7488 & 0.1961 & 1.7325 & 0.7584 \\
\hline 2008 & 484.41 & 2.9274 & 3.0699 & 3.9915 & 2.8725 & 3.0604 \\
\hline 2009 & 533.80 & 3.9316 & 4.1202 & 4.6662 & 4.7076 & 4.1110 \\
\hline 2010 & 626.44 & 1.8714 & 1.7347 & 1.8493 & 0.8526 & 1.7429 \\
\hline 2011 & 717.03 & 5.3555 & 5.3306 & 6.2615 & 4.7901 & 5.3382 \\
\hline 2012 & 723.50 & 3.2189 & 3.0683 & 2.3416 & 2.4921 & 3.0603 \\
\hline MAPE & & 2.4076 & 2.2872 & 2.1920 & 2.2994 & 2.5209 \\
\hline 2013 & 813.30 & 0.7596 & 0.3881 & 2.8641 & 2.1901 & 0.3806 \\
\hline 2014 & 867.24 & 3.4312 & 2.7748 & 1.9197 & 2.8637 & 2.7674 \\
\hline 2015 & 875.37 & 11.9145 & 10.8650 & 4.2162 & 0.5438 & 10.8573 \\
\hline 2016 & 924.89 & 15.4547 & 13.9845 & 5.4247 & 1.9871 & 13.9767 \\
\hline 2017 & 996.55 & 16.5873 & 14.6781 & 4.2545 & 7.7419 & 14.6704 \\
\hline 2018 & 1118.79 & 12.8112 & 10.5247 & 1.3278 & 18.0688 & 10.5175 \\
\hline MAPE & & 10.1598 & 8.8692 & 3.3345 & 5.5659 & 8.8616 \\
\hline
\end{tabular}

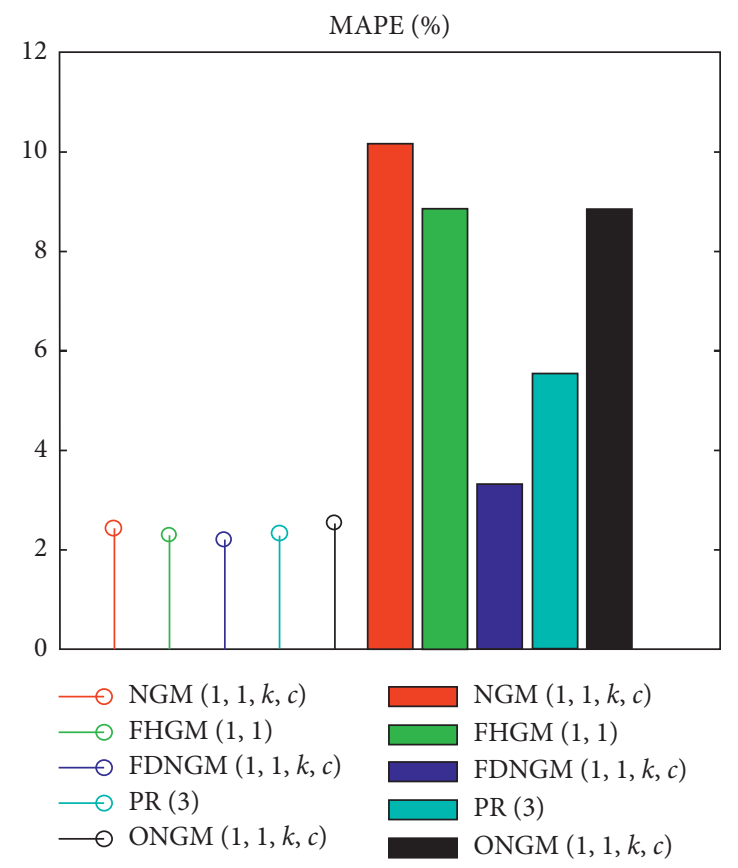

(a)

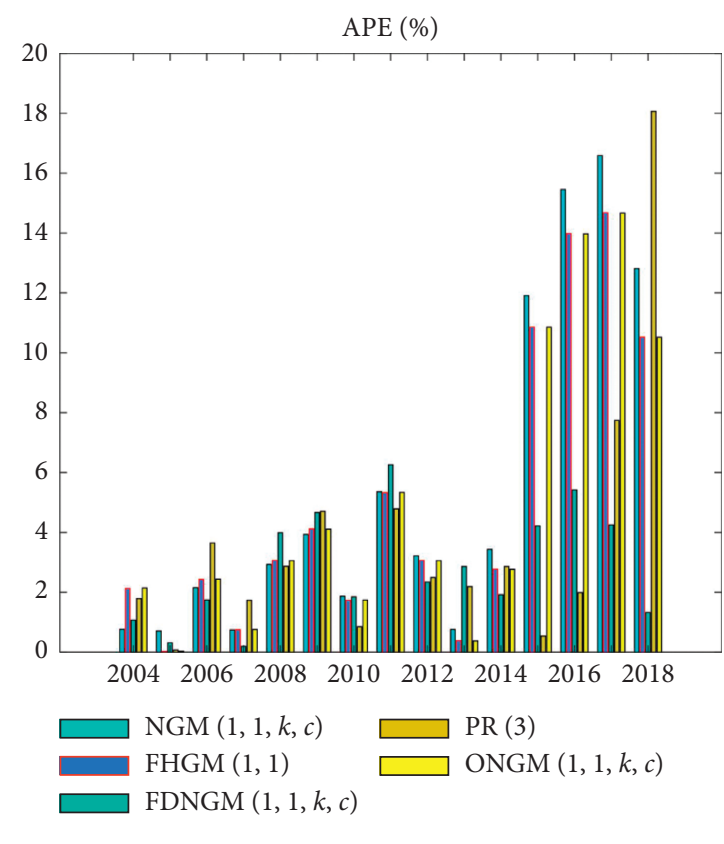

(b)

FIgure 3: APEs and MAPEs of the competitive models in Case 1. 
TABle 4: The error-value indices of the competing models in Case 2.

\begin{tabular}{|c|c|c|c|c|c|c|}
\hline Year & Data & NGM (\%) & FHGM (\%) & FDNGM (\%) & $\mathrm{PR}(3)$ & ONGM (\%) \\
\hline 2001 & 274.30 & 0.0000 & 3.7915 & 0.0000 & 0.1272 & 0.0000 \\
\hline 2002 & 291.84 & 4.9274 & 11.7633 & 0.0012 & 0.2375 & 12.1678 \\
\hline 2003 & 339.08 & 0.3097 & 4.4149 & 1.4094 & 1.4931 & 4.8043 \\
\hline 2004 & 396.72 & 2.5333 & 0.3907 & 0.0003 & 0.0835 & 0.0215 \\
\hline 2005 & 467.63 & 3.6653 & 2.8313 & 1.8665 & 1.9901 & 2.4816 \\
\hline 2006 & 561.41 & 2.1125 & 2.0377 & 1.7288 & 2.0552 & 1.7143 \\
\hline 2007 & 705.23 & 4.4709 & 4.2454 & 3.6870 & 3.3347 & 4.5319 \\
\hline 2008 & 812.94 & 3.1097 & 2.8981 & 1.6931 & 1.5280 & 3.1753 \\
\hline 2009 & 895.20 & 2.4330 & 2.3727 & 3.8457 & 3.5135 & 2.0920 \\
\hline 2010 & 1069.41 & 0.5330 & 1.0726 & 0.0003 & 1.1227 & 1.3341 \\
\hline MAPE & & 2.6772 & 3.5818 & 1.5814 & 1.5486 & 3.5914 \\
\hline 2011 & 1305.30 & 5.7519 & 6.8781 & 6.7281 & 8.8285 & 7.1163 \\
\hline 2012 & 1463.00 & 2.9973 & 4.9200 & 6.1571 & 9.6438 & 5.1568 \\
\hline 2013 & 1705.37 & 4.2114 & 6.9715 & 9.9952 & 14.9601 & 7.1983 \\
\hline 2014 & 1868.94 & 0.4216 & 3.4672 & 8.8931 & 15.8696 & 3.6971 \\
\hline 2015 & 1931.75 & 11.4466 & 5.9433 & 2.9000 & 12.7471 & 5.6952 \\
\hline 2016 & 2078.06 & 18.6741 & 11.4756 & 1.1905 & 14.0055 & 11.2188 \\
\hline 2017 & 2393.70 & 17.8744 & 9.3366 & 6.6300 & 21.7045 & 9.0883 \\
\hline MAPE & & 8.7682 & 6.9989 & 6.0706 & 13.9656 & 7.0244 \\
\hline
\end{tabular}

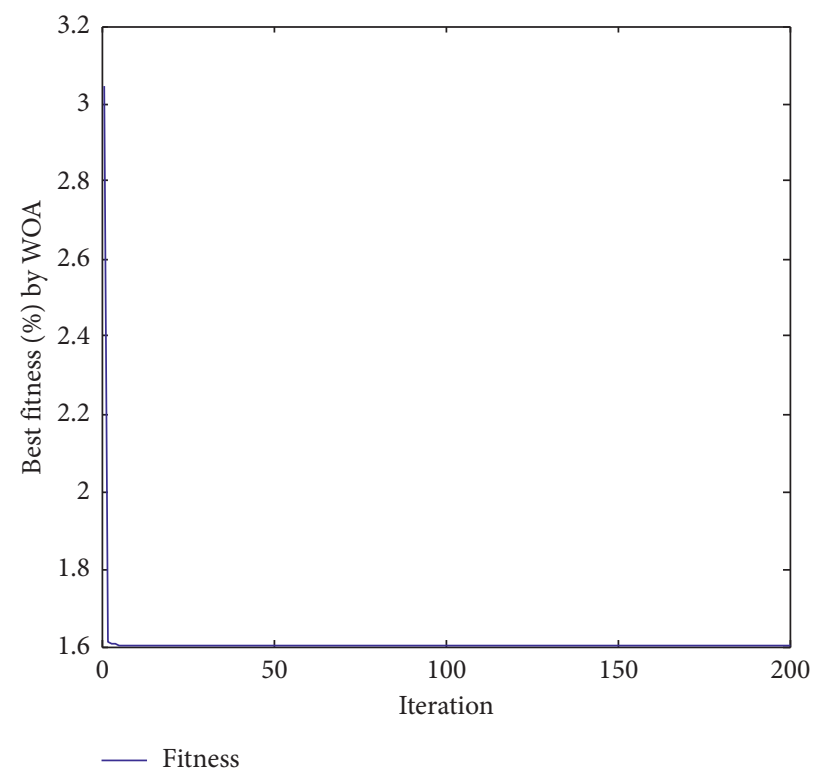

FIGURE 4: Searching process of the WOA-based FDNGM $(1,1, k, c)$ model in Case 2.

TABLE 5: The predicted values of original domain by employing the competing models in Case 2.

\begin{tabular}{ccccccc}
\hline Year & Data & NGM & FHGM & FDNGM & PR(3) \\
\hline 2001 & 274.30 & 274.30 & 284.70 & 274.30 & 274.65 & 274.30 \\
2002 & 291.84 & 277.46 & 257.51 & 291.84 & 334.30 & 33.53 \\
2003 & 339.08 & 338.03 & 324.11 & 396.72 & 396.39 \\
2004 & 396.72 & 406.77 & 398.27 & 476.36 & 476.94 \\
2005 & 467.63 & 484.77 & 480.87 & 571.12 & 572.95 \\
2006 & 561.41 & 573.27 & 572.85 & 679.23 & 681.71 \\
2007 & 705.23 & 673.70 & 675.29 & 799.18 & 800.52 \\
2008 & 812.94 & 787.66 & 789.38 & 929.63 & 926.65 \\
2009 & 895.20 & 916.98 & 916.44 & 1069.41 & 1057.40 \\
2010 & 1069.41 & 1063.71 & 1057.94 & 1217.48 & 1190.06 \\
2011 & 1305.30 & 1230.22 & 1215.52 & & 971.03 \\
\hline
\end{tabular}


TABLE 5: Continued.

\begin{tabular}{|c|c|c|c|c|c|c|}
\hline Year & Data & NGM & FHGM & FDNGM & $\mathrm{PR}(3)$ & ONGM \\
\hline 2012 & 1463.00 & 1419.15 & 1391.02 & 1372.92 & 1321.91 & 1387.56 \\
\hline 2013 & 1705.37 & 1633.55 & 1586.48 & 1534.92 & 1450.24 & 1582.61 \\
\hline 2014 & 1868.94 & 1876.82 & 1804.14 & 1702.73 & 1572.35 & 1799.84 \\
\hline 2015 & 1931.75 & 2152.87 & 2046.56 & 1875.73 & 1685.51 & 2041.77 \\
\hline 2016 & 2078.06 & 2466.12 & 2316.53 & 2053.32 & 1787.02 & 2311.19 \\
\hline 2017 & 2393.70 & 2821.56 & 2617.19 & 2235.00 & 1874.16 & 2611.25 \\
\hline
\end{tabular}

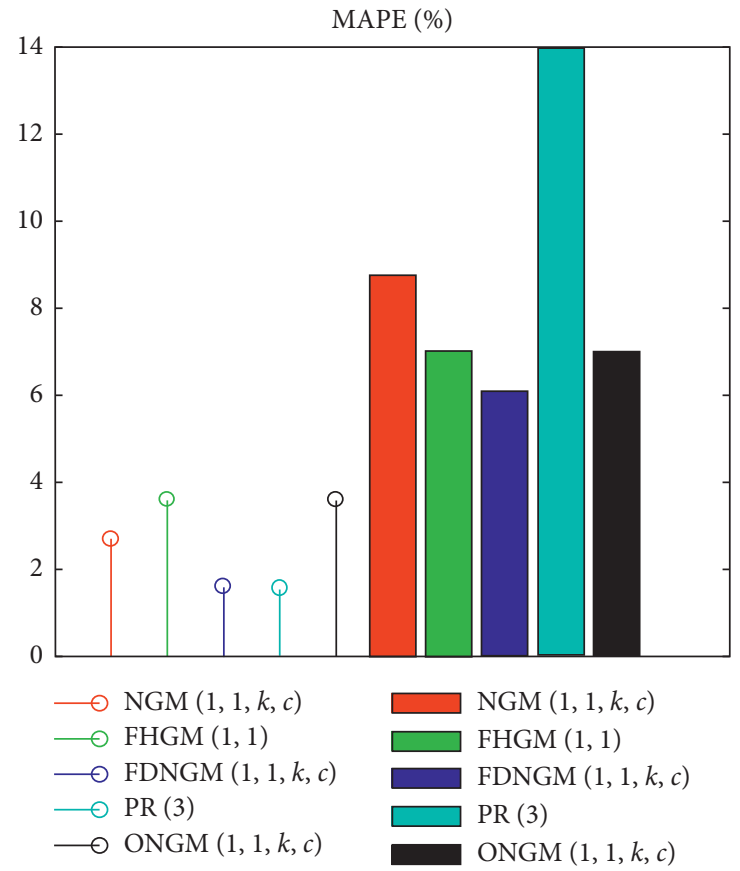

(a)

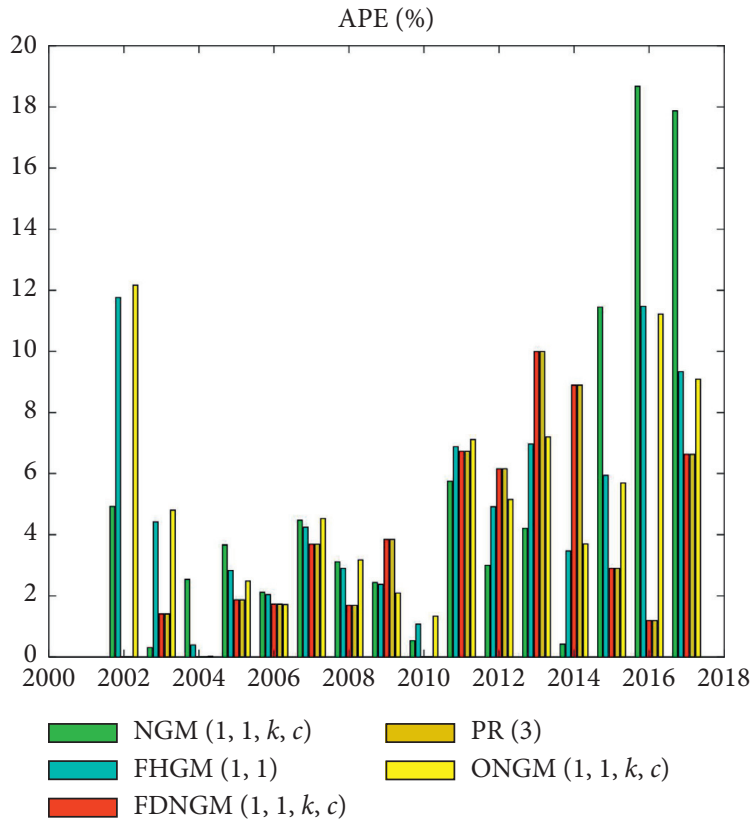

(b)

Figure 5: APEs and MAPEs of the competitive models in Case 2.

\section{Conclusion}

Aiming to improve the prediction precision of the existing $\operatorname{NGM}(1,1, k, c)$ model, this study establishes an optimised $\operatorname{NGM}(1,1, k, c)$ model by combining the ideal of fractional accumulation and dynamic integral median theorem. As a result, a novel model named $\operatorname{FDNGM}(1,1, k, c)$ is proposed thereby. The proposed model is proved to grasp a more flexible and general structure to fit different series by changing the variable of fractional accumulation parameter and dynamic background-value coefficients, thus obtaining a much stronger adaptability in practice. To demonstrate the performance of this approach, we carried out two practical examples to certify the feasibility of the FDNGM $(1,1)$ approach. Additionally, this study presents the dynamic parameters to perfect the background value for the grey modeling technique, applicable for all grey forecasting models.

In addition, we have discussed the superiorities of the proposed FDNGM $(1,1, k, c)$ model; however, it still has some shortcomings, for example, many parameters are contained in the proposed model (elaborated on in Section 3), which brings trouble into the calculation process. How to simplify the model structure will be addressed in our next work.

\section{Data Availability}

The data used to support the findings of this study are included within the article.

\section{Conflicts of Interest}

The authors declare that they have no conflicts of interest.

\section{Acknowledgments}

This work was supported by the National Natural Science Foundation of China (11661001).

\section{References}

[1] J. L. Deng, "Control problems of grey systems," System Control Letter, vol. 1, no. 5, pp. 288-294, 1982.

[2] C. Liu, W.-Z. Wu, W. Xie, T. Zhang, and J. Zhang, "Forecasting natural gas consumption of China by using a novel 
fractional grey model with time power term," Energy Reports, vol. 7, pp. 788-797, 2021.

[3] B. Zeng, M. Zhou, X. Liu, and Z. Zhang, "Application of a new grey prediction model and grey average weakening buffer operator to forecast China's shale gas output," Energy Reports, vol. 6, pp. 1608-1618, 2020.

[4] W. Zhou, X. Wu, S. Ding, and J. Pan, "Application of a novel discrete grey model for forecasting natural gas consumption: a case study of Jiangsu Province in China," Energy, vol. 200, Article ID 117443, 2020.

[5] C. Zheng, W.-Z. Wu, W. Xie, Q. Li, and T. Zhang, "Forecasting the hydroelectricity consumption of China by using a novel unbiased nonlinear grey Bernoulli model," Journal of Cleaner Production, vol. 278, Article ID 123903, 2021.

[6] U. Şahin, "Projections of Turkey's electricity generation and installed capacity from total renewable and hydro energy using fractional nonlinear grey Bernoulli model and its reduced forms," Sustainable Production and Consumption, vol. 23, pp. 52-62, 2020.

[7] C. Liu, W.-Z. Wu, W. Xie, and J. Zhang, "Application of a novel fractional grey prediction model with time power term to predict the electricity consumption of India and China," Chaos, Solitons \& Fractals, vol. 141, Article ID 110429, 2020.

[8] C. Liu, T. Lao, W. Z. Wu, and W. Xie, "Application of optimized fractional grey model-based variable background value to predict electricity consumption," Fractals, vol. 29, no. 2, 2020.

[9] H. Zhao and W. Lifeng, "Forecasting the non-renewable energy consumption by an adjacent accumulation grey model," Journal of Cleaner Production, vol. 275, Article ID 124113, 2020.

[10] S.-L. Lu, "Integrating heuristic time series with modified grey forecasting for renewable energy in Taiwan," Renewable Energy, vol. 133, pp. 1436-1444, 2019.

[11] S. Ding, "A novel discrete grey multivariable model and its application in forecasting the output value of China's hightech industries," Computers \& Industrial Engineering, vol. 127, pp. 749-760, 2019.

[12] L. Zeng, "Analysing the high-tech industry with a multivariable grey forecasting model based on fractional order accumulation," Kybernetes, vol. 48, no. 6, pp. 1158-1174, 2019.

[13] U. Şahin and T. Şahin, "Forecasting the cumulative number of confirmed cases of COVID-19 in Italy, UK and USA using fractional nonlinear grey Bernoulli model," Chaos, Solitons, and Fractals, vol. 138, Article ID 109948, 2020.

[14] Z. Lixia, T. Hong, and H. Miao, "Grey system analysis in the field of medicine and health," Grey Systems: Theory and Application, vol. 9, no. 2, pp. 251-258, 2019.

[15] J. Cui, Y. G. Dang, and S. F. Liu, "Novel grey forecasting model and its modeling mechanism," Control and Decision, vol. 24, no. 11, pp. 1702-1706, 2009.

[16] X. Ma and Z. Liu, "Predicting the cumulative oil field production using the novel grey ENGM model," Journal of Computational and Theoretical Nanoscience, vol. 13, no. 1, pp. 89-95, 2016.

[17] M. Tong, X. Zhou, and B. Zeng, "Optimization of background value in grey $\operatorname{NGM}(1,1, k)$ model," Control and Decision, vol. 32, no. 3, pp. 507-514, 2017.

[18] B. Zeng and S. Liu, "A self-adaptive intelligence gray prediction model with the optimal fractional order accumulating operator and its application," Mathematical Methods in the Applied Sciences, vol. 40, no. 18, pp. 7843-7857, 2017.

[19] L.-F. Wu, S.-F. Liu, W. Cui, D.-L. Liu, and T.-X. Yao, "Nonhomogenous discrete grey model with fractional-order accumulation," Neural Computing and Applications, vol. 25, no. 5, pp. 1215-1221, 2014.

[20] W. Wu, X. Ma, Y. Zhang, W. Li, and Y. Wang, "A novel conformable fractional non-homogeneous grey model for forecasting carbon dioxide emissions of BRICS countries," Science of the Total Environment, vol. 707, Article ID 135447, 2020.

[21] L. Wu, S. Liu, L. Yao, S. Yan, and D. Liu, "Grey system model with the fractional order accumulation," Communications in Nonlinear Science and Numerical Simulation, vol. 18, no. 7, pp. 1775-1785, 2013.

[22] C. Liu, W. Xie, T. Lao et al., "Application of a novel grey forecasting model with time power term to predict China's GDP," Grey Systems Theory and Application Ahead-of-Print, vol. 93, 2020.

[23] S. Mao, M. Gao, X. Xiao, and M. Zhu, "A novel fractional grey system model and its application," Applied Mathematical Modelling, vol. 40, no. 7-8, pp. 5063-5076, 2016.

[24] X. Ma, X. Mei, W. Wu, X. Wu, and B. Zeng, "A novel fractional time delayed grey model with Grey Wolf Optimizer and its applications in forecasting the natural gas and coal consumption in Chongqing China," Energy, vol. 178, pp. 487-507, 2019.

[25] C. Liu, W. Xie, W.-Z. Wu, and H. Zhu, "Predicting Chinese total retail sales of consumer goods by employing an extended discrete grey polynomial model," Engineering Applications of Artificial Intelligence, vol. 102, no. 3, Article ID 104261, 2021.

[26] X. Zhu, Y. Dang, and S. Ding, "Using a self-adaptive grey fractional weighted model to forecast Jiangsu's electricity consumption in China," Energy, vol. 190, Article ID 116417, 2020.

[27] Y. Chen, W. Lifeng, L. Lianyi, and Z. Kai, "Fractional Hausdorff grey model and its properties," Chaos, Solitons \& Fractals, vol. 138, Article ID 109915, 2020.

[28] X. Ma, W. Wu, B. Zeng, Y. Wang, and X. Wu, "The conformable fractional grey system model," ISA Transactions, vol. 96, pp. 255-271, 2020.

[29] S. Li, B. Zeng, X. Ma, and D. Zhang, "A novel grey model with A three-parameter background value and its application in forecasting average annual water consumption per capita in urban areas along the yangtze river basin," Journal of Grey System, vol. 32, no. 1, 2020.

[30] W. Zhang, R. Xiao, B. Shi, H.-h. Zhu, and Y.-j. Sun, "Forecasting slope deformation field using correlated grey model updated with time correction factor and background value optimization," Engineering Geology, vol. 260, Article ID 105215, 2019.

[31] S. Mirjalili and A. Lewis, "The whale optimization algorithm," Advances in Engineering Software, vol. 95, pp. 51-67, 2016.

[32] G. Xiong, J. Zhang, D. Shi, and Y. He, "Parameter extraction of solar photovoltaic models using an improved whale optimization algorithm," Energy Conversion and Management, vol. 174, pp. 388-405, 2018.

[33] M. A. E. Aziz, A. A. Ewees, and A. E. Hassanien, "Whale optimization algorithm and moth-flame optimization for multilevel thresholding image segmentation," Expert Systems with Applications, vol. 83, pp. 242-256, 2017.

[34] Z. Alameer, M. A. Elaziz, A. A. Ewees, H. Ye, and Z. Jianhua, "Forecasting gold price fluctuations using improved multilayer perceptron neural network and whale optimization algorithm," Resources Policy, vol. 61, pp. 250-260, 2019.

[35] B. Chen, Polynomial Regression, Springer Texts in Statistics, Berlin, Germany, 1986.

[36] Z. Liu, Y. G. Dang, and L. Wei, “Optimization of background value and time response function in $\operatorname{NGM}(1,1, \mathrm{k})$ control and decision," 2016. 